\title{
The Influence of Inclusions on Low Cycle Fatigue Life in a P/M Nickel-Base Disk Superalloy
}

\author{
Eric S. Huron \\ Paul G. Roth \\ General Electric Aircraft Engines (GEAE) \\ Cincinnati, OII 45215
}

\begin{abstract}
$\underline{\text { Abstract }}$
The high alloy content of advanced nickel-base disk superalloys calls for powder metallurgy $(\mathrm{P} / \mathrm{M})$ processing to minimize segregation produced by conventional cast \& wrought processing. Although the technology has developed to allow reliable application of $\mathrm{P} / \mathrm{M}$ materials, of concern are ceramic inclusions which are intrinsic to the process due to the use of ceramic crucibles in producing the raw meltstock and in the powder atomization process itself.
\end{abstract}

For robust disk design, the impact of ceramics on Low Cycle Fatigue (LCF) must be assessed. Actual production material can be evaluated but the low frequency of larger inclusions means that impracticably large volumes of material must be tested. To address this problem, tests were run on powder seeded with controlled distributions of ceramics. The alloy was the current GEAE disk material Rene' 88 DT (R'88DT). Two types of ceramic seeds were added at two size/density combinations. Small seeds $(-270 /+325$ mesh) were added to -270 mesh powder al rates (numbers per unit volume) predicted via probabilistic calculations to cause surface initiations. Large seeds $(-80 /+100$ mesh) were added to the powder at rates predicted to cause internal initiations. The powder was consolidated and processed using production parameters. LCF tests were made on samples taken fronn fully heat treated forgings at $204^{\circ} \mathrm{C}$ and $649^{\circ} \mathrm{C}$ at two stress levels. Roughly half of the bars were shotpeened to study the ability of this processing to suppress surface initiations.

'The impact of the seeds was significant and was a function of seed type and size, temperature, and bar surface condition. At $204^{\circ} \mathrm{C}$ life decreased with increasing secd size up to a maximum life reduction of $33-50 \%$. Shotpeening only slightly improved the lives of small seeded bars. At $649^{\circ} \mathrm{C}$, the seeds had dramatic impacts. Small seeds reduced life by 1-2 orders of magnitude and large seeds by a further 1-2 orders of magnitude. Shotpeening did suppress surface initiations and significantly improved life. The relative impacts between $204^{\circ} \mathrm{C}$ and $649^{\circ} \mathrm{C}$ and the impact of shotpeening depended on failure mechanisms -- at $204^{\circ} \mathrm{C}$ most of the failures initiated at facets, explaining a relatively minor seeding impact at this temperature while at $649^{\circ} \mathrm{C}$ almost all failures initiated at the seeds (or at intrinsic inclusions in the unseeded material).
The results of this testing have been used to develop an incubation model incorporated in GEAE's Probabilistic Fracture Mechanics (PFM) methodology used for design life calculations.

\section{Introduction}

Advanced nickel-base superalloys for disk applications are highly alloyed to meet design strength and temperature requirements. Given the high alloy content of these materials, powder metallurgy $(\mathrm{P} / \mathrm{M})$ processing is preferred over conventional cast \& wrought processing to prevent excessive segregation.

Ceramic inclusions are inherent in the $\mathrm{P} / \mathrm{M}$ process. Inclusions are introduced from the refractory crucible material used to produce the starting Vacuum Induction Melt (VIM) ingot, the secondary VIM crucible used in the powder atomization, the nozzle and guide tube arrangement for the atomization stream, and by reactions between the molten stream and the gases in the atomization chamber.

The impact of ceramics on fatigue life must be quantified. Historically this has been attempted through LCF tests on actual production forgings, but inclusion content is seldom adequately characterized and varies from one forging lot to the next, and to capture larger inclusions, large volumes of material must be tested; also, many tests fail at parent metal initiation sites with no inclusion present. For all these reasons, prohibitively large numbers of LCF tests are required, and interpretation of the resulting data is usually difficult and controversial.

Testing of intentionally seeded material offers significant advantages. The impact of a much larger range of inclusion sizes may be assessed. Seeding distributions may be statistically described, and competition with grain initiated failures may be managed by adjustment of seeding rates.

This paper presents a seeded LCF study of P/M R'88DT, GEAE's current advanced disk alloy (References 1,2). Loose powder was doped with controlled distributions of two types of ceramic seeds. The seeded powder was consolidated via cxtrusion, isothermally forged into pancakes, and heat treated. LCF bars were taken from the pancakes and tested. 
Table I. Composition of R'88DT (Weight Percent)

$\begin{array}{ccccccccccc}\text { Co } & \text { Cr } & \text { Mo } & \text { W } & \text { Al } & \text { Ti } & \text { Nb } & \text { B } & \text { C } & \text { Zr } & \text { Ni } \\ 13 & 16 & 4 & 4 & 2.1 & 3.7 & 0.7 & 0.015 & 0.03 & 0.03 & \text { bal }\end{array}$

The study focused on three points in question:

- The effect of small inclusions inherent in powder screened to -270 mesh

- The effect of larger inclusions representative of an unexpected contamination event.

- The influence of shotpeening on surfacc crack initiation at inclusions.

Portions of the program were performed with the assistance of SNECMA at the Gennevilliers, France facility.

\section{Procedure}

R'88DT powder obtained through GEAE's normal production sources was screened to -270 mesh. The chemistry is given in Table I.

The -270 mesh size was chosen to limit the size of naturally occurring inclusions in the material to better assess the intluence of the added ceramic seeds. Two types of seeds were added, termed Type 1 and Type 2, consistent with past studies of inclusion behavior (Reference 3). A hard, blocky seed (Type 1) was added to represent crystalline or glassy, non-deforming ceramics, such as might occur due to breakage of an alumina crucible. The Type 1 was simulated using an $\mathrm{Al}_{2} \mathrm{O}_{3} / \mathrm{MgO}$ casting firebrick mold material supplied by SNECMA. A soft, deformable, agglomerate seed (Type 2) was added to represent reactive inclusions originating from patching putties or furnace assembly cements, or by reaction of the molten strean with trace gasses in the atomization chamber. The Type 2 was simulated by a moist $\mathrm{Al}_{2} \mathrm{O}_{3}$-based paste with a phosphorous/silicon-containing binder, sold under the trade name RAM 90 by Combustion Engineering Refractories. The RAM 90 was given the recommended drying cycle of $54^{\circ} \mathrm{C} / 5.5 \mathrm{hrs}+110^{\circ}$ $\mathrm{C} / 16.5 \mathrm{hrs}$, then fired at $1149^{\circ} \mathrm{C} / 4 \mathrm{hrs} /$ furnace cool. Both seed types were crushed in a mortar and pestle and sieved to appropriate distributions as discussed below.

Blending and extrusion experiments were made to verify the suitability of the Type 2 seed material. Carefully weighed amounts of sieved RAM 90 were added to powder samples of slightly finer mesh size; the samples were aggressively blended and then sieved to recapture the RAM 90. High recoveries showed the blending did not cause excessive disintegration of the ceramic. In addition, a laboratory extrusion using a $8.6 \mathrm{~cm}$ dia. $x 15.24 \mathrm{~cm}$ long can was filled with -200 mesh R'95 powder with a few grams of $-80 /+100$ and $-170 /+325$ RAM 90 material. After extrusion at $1066^{\circ} \mathrm{C}$, metallography (Figure 1), showed that the RAM 90 inclusions had the desirable characteristics of a Type 2: a multi-particle makeup with a tendency to elongate in the direction of deformation.

Control of seeding rates presented a challenge. Given that exact counts of hundreds of thousands of seeds are not practical, seeds

were added by weight. Several means were used by SNECMA to obtain average seed weights, including: measurements of samples of 1000 large seeds and 10000 small seeds; use of a Coulter counter to measure size of individual particles; and computations based on assumed ellipsoidal shapes and mean diameter measurements. Multiple trials with the various methods suggested that the count-plus-weigh method was most accurate.

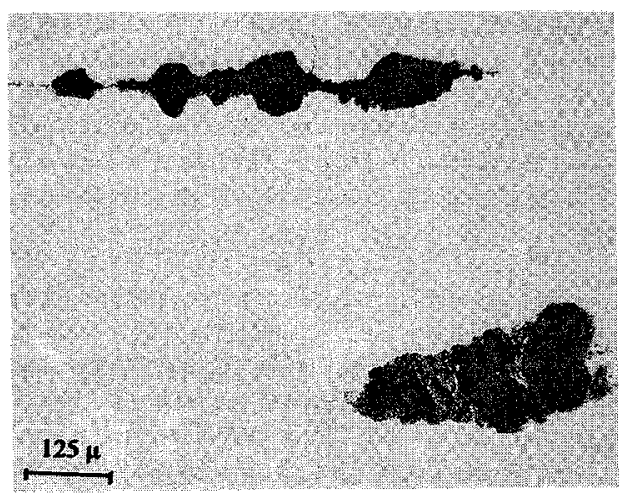

Figure 1: Appearance of RAM-90 material used to simulate Type 2 seeds after extrusion. The elongation of the seeds parallel with the deformation direction resembles that of intrinsic Type 2 inclusions found in production powder.

Two seed sizes were used. Small mesh seeds, at $680 / \mathrm{kg}$ goal density, were intended to represent the upper end of the distribution that would normally be present in -270 mesh powder, but at an artificially high frequency to promote a high percentage of surface intersections. Large $-80 /+100$ mesh seeds, at $36 / \mathrm{kg}$ goal density, were intended to represent inclusions arising from contamination.

The general seeding procedure was to add a weighed quantity of ceramic to $5 \mathrm{~kg}$ of powder, mix for four hours in a Turbula blender, then pour the batch into an extrusion can. This was repeated 8 times to end with $40 \mathrm{~kg}$ in the can. After filling, the can was evacuated over 24 hours using a diffusion pump system attached to the fill tube, then sealed by crimping the tube.

Eight extrusions were made: one baseline (unseeded), two seeded with small Type 1 seeds, two seeded with small Type 2 seeds, one seeded with large Type 1 seeds, and one seeded with large Type 2 seeds. SNECMA was responsible for compaction and extrusion of the powder, sectioning into mults, and performing the isothermal forging. Stainless extrusion cans of $19 \mathrm{~cm}$ diameter $\times 28.5 \mathrm{~cm}$ long were compacted and extruded at a 5.5:1 reduction ratio at $1052^{\circ} \mathrm{C}$. 
Each extrusion yiclded a total of five mults. The mults were isothermally forged using a laboratory isothermal press at $1050^{\circ} \mathrm{C}$. Forgings were heat treated through a $1149^{\circ} \mathrm{C}$ supersolvus solution followed by air cooling. Pancakes were equipped with mid-thickness thermocouples and the interior cooling rates ranged from $79^{\circ} \mathrm{C} / \mathrm{min}$ for the mid-radius to $88^{\circ} \mathrm{C} / \mathrm{min}$ near the rim.

The material was compared to production R'88DT and found to be representative in microstructure (ASTM 7/8 ALA 4 grain size), tensile strength (1062 $\mathrm{MPa} 0.2 \%$ yield strength and $1524 \mathrm{MPa}$ ultimate at $204^{\circ} \mathrm{C}, 1007 \mathrm{MPa}$ yield strength and $1503 \mathrm{MPa}$ ultimate at $649^{\circ} \mathrm{C}$ ), and creep strength (155 hours to $0.2 \%$ at $704^{\circ}$ $\mathrm{C} / 690 \mathrm{MPa}$ ).

Eight LCF specimens were machined from each $18 \mathrm{~cm}$ diameter by $2.5 \mathrm{~cm}$ thick pancake (Figure 2). The LCF specimen gage section was relatively low in aspect ratio, $1.016 \mathrm{~cm}$ diameter by $1.905 \mathrm{~cm}$ tall (Figure 3), to help promote internal failures, with large radii from shoulder to gage to minimize stress concentration. Welded IN718 shanks and button heads were used. Roughly half of the test bars were shotpeened by $\mathrm{GE}$ using $\mathrm{CCW} 31$ shot at $7 \mathrm{~A}$ intensity and $125 \%$ coverage. Strain-controlled 0 -max-0 LCF tests were performed at two strain levels at $204^{\circ} \mathrm{C}(0.52 \%$ and $0.70 \%)$ and two strain levels at $649^{\circ} \mathrm{C}(0.60 \%$ and $0.80 \%)$. The first 100 cycles of the test were done using a specialized procedure to minimize serrated yielding (Reference 4 ). This procedure utilized a triangle waveform in strain control at $1.5 \mathrm{cpm}$ coupled with a $0.02 \%$ increase in strain range. $A$ fter the first 100 cycles, the next 24 hours were run at the target strain range in strain control at 30 cpm, and any cycles over the first 24 hours (about 42,000 cycles) were conducted in load control at $300 \mathrm{cpm}$. After testing fractography of the bars was performed using the SEM. Inclusions and grain facet initiation sites were characterized for size, type, and location.

The final matrix covered two temperatures, two target stress levels (achieved via strain control) at each temperature, two seed types, two seed sizes, and peened and unpeened surface finish as variables (refer to Table II).

\section{Results}

Post-test fractography showed that the chosen ceramics were reasonably effective in simulating Type 1 and 2 inclusions found in production material. The median initiation site sizes (based on boxed areas) were 4,770 square microns for the small seeds and 40,000 square microns for the large seeds. These values fell below the median sizes of the loose seeds due to inclusion orientation relative to the stress plane of the specimens. Baseline material failed at grains or at intrinsic inclusions with an average size of about 2,130 square microns.

At low temperature, some seeded bars failed at grain facets indicating a competition between ceramics and grain facets for crack initiation. The influence of seeding rates on observed behavior are further discussed below. Figure 4 presents a comparison between typical initiation sites at grain facets, intrinsic inclusions, Type 1 seeds, and Type 2 seeds.

Post-test metallographic study was conducted on ten small sceded specimens looking for evidence of secondary cracking. Significant

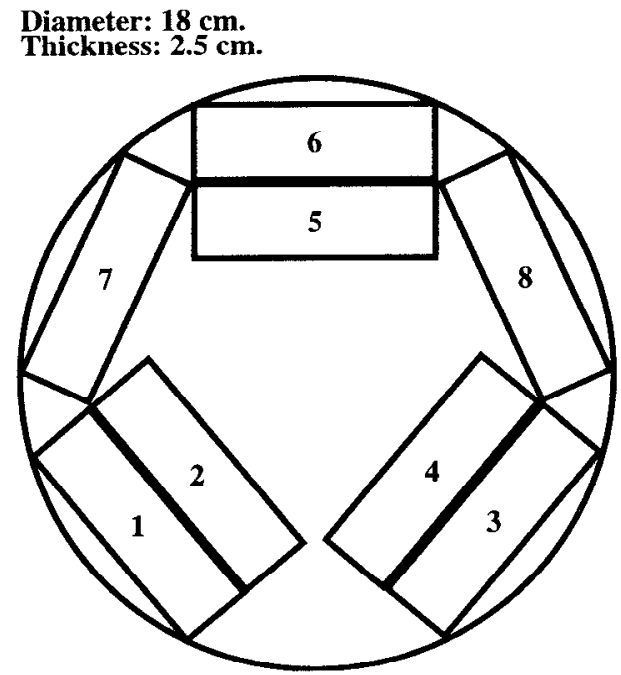

Figure 2: Specimen sectioning plan for subscale pancakes. The tangential layout of the specimens was used to provide a consistent deformation history at the gage section of the bars. The test matrix was blocked against the inner row position vs. the outer row position to ascertain whether location had any statistical impact.

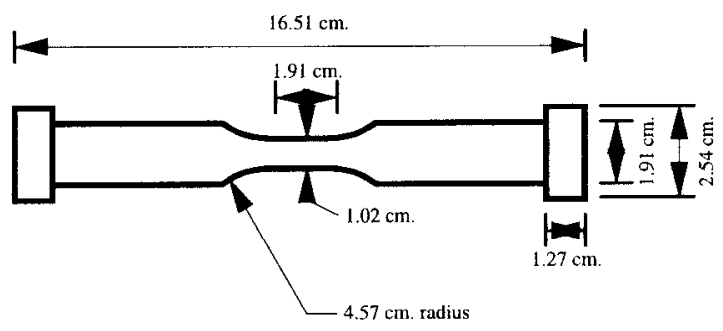

Figure 3: Low Cycle Fatigue specimen design.

numbers of secondary grain-initiated cracks were found in speci mens tested at $204^{\circ} \mathrm{C}$ and very little secondary cracking in specimens tested at $649^{\circ} \mathrm{C}$ (Figure 5). Also, it was found that inclusions were most often tangentially oriented in the pancake forgings if oriented at all. This countered conventional wisdom which held that they would be oriented radially.

Given a distribution of inclusion areas, a distribution of aspect ratios, a seeding rate, the diameter and length of a cylinder, and the assumption of tangential orientation, it is possible to estimate by Monte Carlo simulation the expected distribution of the largest inclusion area normal to the stress field in a test bar volume. The estimated distribution should approximate the observed distribution of initiation areas if (as is expected when all failures are subsurface) the specimens tend to fail from the largest contained inclusions. 


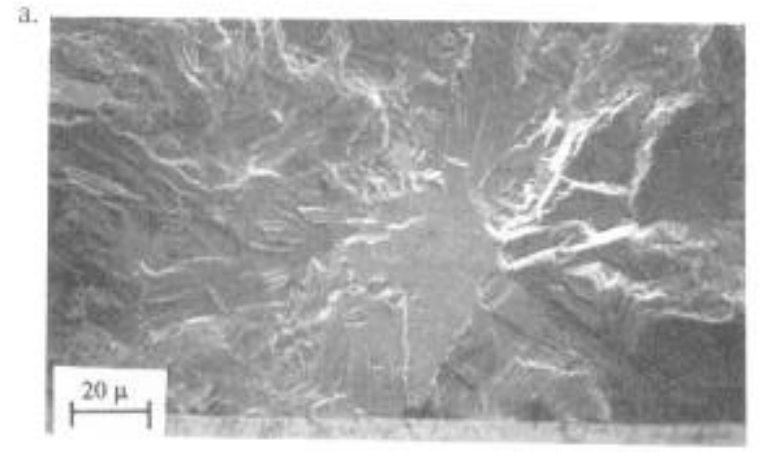

b.

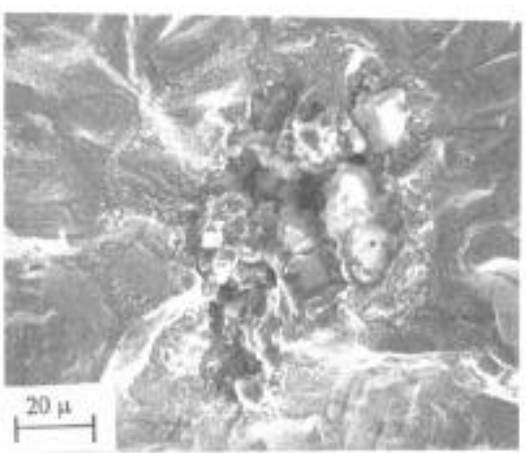

c.

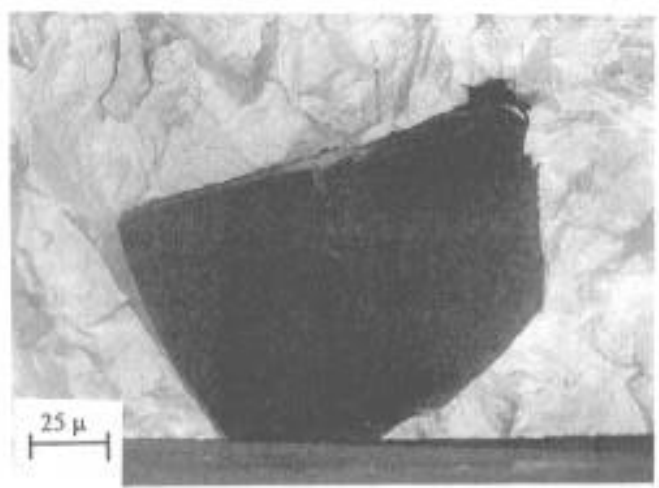

d.

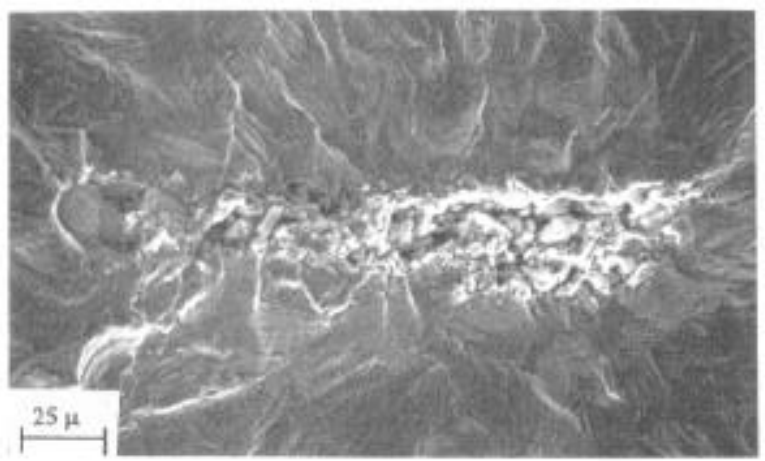

Figure 4: Micrographs of typical initiation sites, a) Grain facet initiation. b) Intrinsic inclusion in unseeded baseline material. c) Typical Type I seed. d) Typical Type 2 seed.
Application to the small and large seeding distributions with seeding rates of 27 inclusions per cubic centimeter for the small seeded material and 5 inclusions per cubic centimeter for the large yielded the comparisons shown in Figure 6.

Note that size is plotted against the probability of exceeding that size. Exceedance probability is estimated for experimental data by ranking the data from largest to smallest, then using the expression

$$
(n-1+0.5) / n
$$

where $n$ is the number of points and $i$ is the ranking of the $i$ th data point.

The impact of the seeds was significantly different from the $204^{\circ}$ $C$ tests to the $649^{\circ} \mathrm{C}$ tests. Figure 7 summarizes the Type I data compared to baseline unseeded data at $204^{\circ} \mathrm{C}$ (Table II). Note that none of the large Type I seeded bars were peened. There was some spread between large seed and small seed data, but overall the lives were remarkably similar. The lowest lives were for unpeened Large Type I seeded bars. A near surface inclusion of 477 micron length by 191 micron width $(91,107$ square microns boxed area) located gave the lowest life at high stress. At low stress the lowest life was due to a much smaller seed ( $124 \times 102$ microns) unfavorably oriented on the surface.

Figure 8 summarizes the Type 2 data at $204^{\circ} \mathrm{C}$ (Table II). Note that all of the large Type 2 seeded bars were peened. The low lives of these bars were slightly above the lowest lives for the unpeened large Type I seeded bars. At high stress unpeened small seeds located on the surface ( $208 \times 43$ microns and $107 \times 41$ microns boxed areas) gave lives as low as peened large seeds located on the surface $(460) \times 140$ microns and $201 \times 104$ microns boxed areas). At low stress only one peened large seeded bar failed at the surface, and resulted in the lowest life. A very large seed ( $1234 \times 584$ microns boxed area) gave the longest life at low stress, but was located internally. For the small seeded unpeened bars, the Type 2 appeared to have a slightly more negative effect on life than the Type 1. Overall, the peened lives were grouped tightly together and were slightly improved over unpeened lives at high stress due to suppression of lower life surface or near surface seed failures. Most failures were at grains, and although peening changed the location of the grain failures from surface to internal, the life was not dramatically enhanced by peening. An overall comparison of surface vs, internal initiations did show that internal initiation is associated with longer life.

Figures 9 and 10 provide the results at $649^{\circ} \mathrm{C}$ (Table II). Note the wide degree of scatter compared to $204^{\circ} \mathrm{C}$. The relative life impact was much larger. It did not take a particularly large seed to cause a low life at $649^{\circ} \mathrm{C}$. In Figure 9, an unpeened small seeded bar with a planimeter area of only $74 \times 51$ microns fell well below the unseeded baseline. At this same range of stress on the plot, the lives for the large seeded bars tended to fall in order of seed size 3776 cycles for $376 \times 152$ microns, 5326 cycles for $239 \times 122$ microns, and 6126 cycles for $127 \times 86$ microns, while an internal seed of $584 \times 147$ microns gave a life of 17,038 cycles. At the low stress, however, surface failures due to $234 \times 86$ micron and $279 \times$ 121 micron seeds gave very low lives. For the Type 2 seeds (Figure 10), which were all peened, most of the large seeded bars failed internally, but the lives were still low. For most of the data, peened lives were similar to unpeened lives, but there were a few 


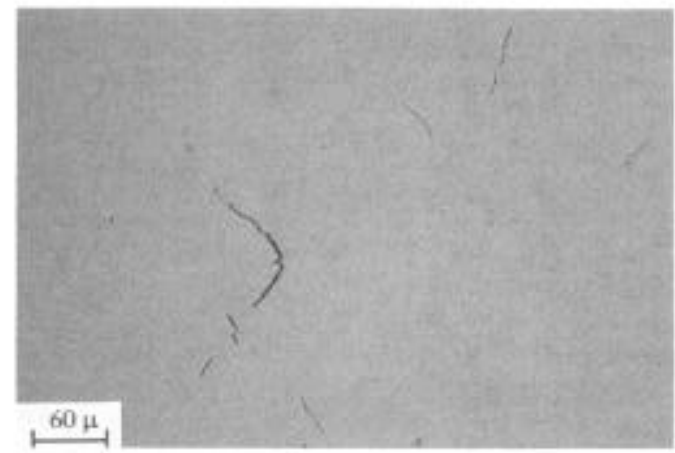

$649^{\circ} \mathrm{C}$

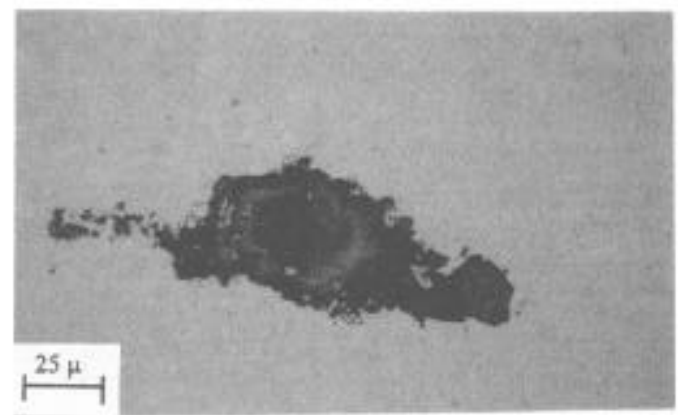

Figure 5: Comparison of secondary cracking in $204^{\circ} \mathrm{C}$ and $649^{\circ}$ C specimens. The $204^{\circ} \mathrm{C}$ specimens had numerous grain-initiated cracks competing to failure while the $649^{\circ} \mathrm{C}$ specimens displayed very little secondary cracking.

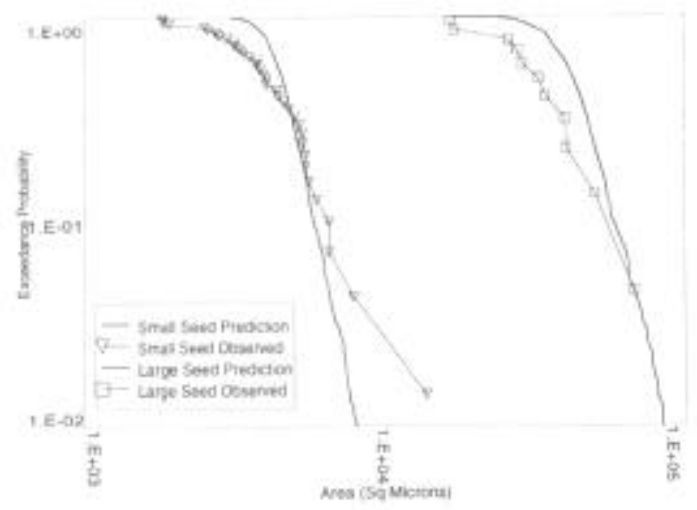

Figure 6: Input size distributions for small and large seeds. Type 1 and Type 2 distributions were nearly equivalent for each seed size.

low lives shown by unpeened seeded bats. Peening was apparently able to prevent these occasional surface failures, but if a bar was inclined to fail internally, obviously peening provided little benefit.

For the Type I seeds, very low lives were observed for surface failures from the unpeened large seeded bars. If the large seeds happened to be located internally, the LCF lives were not as strongly affected. Two low lives were also observed from surface failures at high stress for unpeened small seeded bars. Note that for the Type 2 seeds, most of the failures were internal, but even internally located seeds caused significant life reductions. When the unpeened large Type 1 and peened large Type 2 dati were considered together, peening appeared to have a slightly beneficial effect at high stress because it prevented very low lives due to surface failures.

Some large seeded bars failed at grains for R'88DT (even at $649^{\circ}$ C), which indicated either that grains were more damaging than seeds, or perhaps that given the low seeding density, no seeds were present in the gage section of those particular bars.

At $204^{\circ} \mathrm{C}$, the large Type 1 vs. large Type 2 seeds did show some differences in grain vs, inclusion failures. The two lowest lives at both high and low stress for the large Type 2 seeds were surface failures, even though the bars were peened. This suggested that the nominal 40,000 square micron seed size was above a ceiling of peening protection. In fact, at the low stress the lowest life for the large peened Type 2 was due to a seed of only $406 \times 51$ micron. A survey of the data found that for large Type 1 bars, over $70 \%$ of the bars failed at the surface, while for the unpeened large Type 2 bars, only about $15 \%$ failed at the surface. For the unpeened large Type 1 bars, over $60 \%$ failed at grains, while for the large Type 2 bars, only about $25 \%$ failed at grains.

In general the Type I seeds were very representative of Type I seeds intrinsically present in $\mathrm{P} / \mathrm{M}$ superalloy powder, but the Type 2 seeds gave mixed results. Some showed classic Type 2 behavior, but many looked like Type 1 or mixed Type $1 /$ Type 2 . Jablonski (Reference 5) used $\mathrm{Al}_{2} \mathrm{O}_{3}$ and $\mathrm{SiO}_{2}$ seeds in a study on the effect of inclusions in Low Carbon (LC) Astroloy. The seeds were not described as Type 1 and Type 2, but fractography showed that the $\mathrm{Al}_{2} \mathrm{O}_{3}$ seeds were non-reactive (Type 1) and the $\mathrm{SiO}_{2}$ seeds reacted with the matrix and formed a "granular" inclusion. Therefore $\mathrm{SiO}_{2}$ may provide a better simulation of Type 2 inclusions than the RAM 90. It has been suggested that many Type 2 's in production involve interactions between the atomization nozzle and the metal stream (Reference 3 ). If this is true, more realistic simulation of Type 2 seeds will be difficult.

\section{Discussion}

GEAE's traditional lifing approach, based on LCF curves, is evolving for fracture critical powder metal components by incorporating Probabilistic Fracture Mechanics (PFM) analysis. Supporting this move is a growing validation database which convincingly demonstrates that probabilistics work given the right inputs:

Figure 11 shows comparisons between PFM-predicted and observed failure distributions for five specimen sets: Cylindrical specimens from this study and hourglass specimens from a USAF-funded program (Reference 6); unseeded baseline, large seeded and small seeded; all peened and all tested at the same conditions of strain and temperature. The comparisons demonstrate the capability of the PFM model over the range of probabilities 0.01 to 0.99 when provided the right inputs of inclusion distribution and behavior. It is easily accepted that were the rate of flaw occurrence orders of magnitude lower than the selected seeding rates (i.e., representative of actual production powder cleanliness), the failure probabilities would be proportionately lower in the absence of other failure mechanisms. 
Table II. Test Matrix and Summaries of Observed Lives and Failure Origins

\begin{tabular}{|c|c|c|c|c|c|c|c|c|c|c|c|c|c|c|c|}
\hline & & & & & & & & & & & & & Near & & \\
\hline Test & Seeding & & & Number & Minimum & Maximum & Median & Grain & Inclusion & Other & Percent & Surface & Surface & Internal & Percent \\
\hline Temp & Parameters & Peened & Stress & of Bars & Life & Life & Life & Origins & Origins & Origins & Grains & Origins & Origins & Origins & Surface \\
\hline \multirow[t]{16}{*}{$204 \mathrm{C}$} & Baseline & Yes & High & 15 & 67480 & 83808 & 83808 & 15 & 0 & 0 & $100 \%$ & 0 & 1 & 14 & $0 \%$ \\
\hline & & & Low & 15 & 267084 & 360842 & 317617 & 14 & 0 & 1 & $93 \%$ & 1 & 0 & 14 & $7 \%$ \\
\hline & & No & High & 5 & 48180 & 64017 & 52838 & 5 & 0 & 0 & $100 \%$ & 5 & 0 & 0 & $100 \%$ \\
\hline & & & Low & 4 & 247822 & 302506 & 263565 & 4 & 0 & 0 & $100 \%$ & 4 & 0 & 0 & $100 \%$ \\
\hline & Type I Small & Yes & High & 15 & 69385 & 85067 & 75201 & 14 & 1 & 0 & $93 \%$ & 1 & 1 & 13 & $7 \%$ \\
\hline & $(680 / \mathrm{kg})$ & & Low & 15 & 267530 & 358258 & 304016 & 15 & 0 & 0 & $100 \%$ & 0 & 0 & 15 & $0 \%$ \\
\hline & & No & High & 4 & 43086 & 59964 & 51080 & 4 & 0 & 0 & $100 \%$ & 3 & 1 & 0 & $75 \%$ \\
\hline & & & Low & 5 & 240091 & 317258 & 276005 & 4 & 1 & 0 & $80 \%$ & 5 & 0 & 0 & $100 \%$ \\
\hline & Type 2 Small & Yes & High & 14 & 72526 & 94648 & 79473 & 14 & 0 & 0 & $100 \%$ & 0 & 0 & 14 & $0 \%$ \\
\hline & $(680 / \mathrm{kg})$ & & Low & 15 & 258918 & 357909 & 312821 & 15 & 0 & 0 & $100 \%$ & 0 & 0 & 15 & $0 \%$ \\
\hline & & No & High & 5 & 29284 & 54050 & 52453 & 1 & 4 & 0 & $20 \%$ & 5 & 0 & 0 & $100 \%$ \\
\hline & & & Low & 5 & 216456 & 305988 & 237117 & 5 & 0 & 0 & $100 \%$ & 5 & 0 & 0 & $100 \%$ \\
\hline & Type 1 Large & No & High & 9 & 26703 & 59074 & 45677 & 6 & 3 & 0 & $67 \%$ & 7 & 1 & 1 & $78 \%$ \\
\hline & $(36 / \mathrm{kg})$ & & Low & 8 & 87513 & 305186 & 189908 & 5 & 3 & 0 & $63 \%$ & 7 & 1 & 0 & $88 \%$ \\
\hline & Type 2 Large & Yes & High & 10 & 34451 & 91644 & 77589 & 2 & 8 & 0 & $20 \%$ & 2 & 1 & 7 & $20 \%$ \\
\hline & $(36 / \mathrm{kg})$ & & Low & 10 & 114809 & 350287 & 276678 & 3 & 7 & 0 & $30 \%$ & 1 & 1 & 8 & $10 \%$ \\
\hline \multirow[t]{16}{*}{$649 \mathrm{C}$} & Baseline & Yes & High & 15 & 161661 & 590762 & 291091 & 0 & 15 & 0 & $0 \%$ & 0 & 0 & 15 & $0 \%$ \\
\hline & & & Low & 15 & 520998 & 9529000 & 3926400 & 0 & 15 & 0 & $0 \%$ & 0 & 0 & 6 & $0 \%$ \\
\hline & & No & High & 6 & 244058 & 447111 & 357255 & 0 & 6 & 0 & $0 \%$ & 0 & 0 & 6 & $0 \%$ \\
\hline & & & Low & 3 & 430022 & 2734736 & 2513086 & 0 & 3 & 0 & $0 \%$ & 0 & 0 & 3 & $0 \%$ \\
\hline & Type I Small & Yes & High & 15 & 52723 & 115550 & 81277 & 0 & 15 & 0 & $0 \%$ & 0 & 1 & 14 & $0 \%$ \\
\hline & $(680 / \mathrm{kg})$ & & Low & 14 & 158024 & 628103 & 292015 & 0 & 14 & 0 & $0 \%$ & 0 & 0 & 14 & $0 \%$ \\
\hline & & No & High & 5 & 8006 & 124028 & 58768 & 0 & 4 & 1 & $0 \%$ & 2 & 0 & 3 & $40 \%$ \\
\hline & & & Low & 5 & 130027 & 605028 & 276926 & 0 & 5 & 0 & $0 \%$ & 0 & 1 & 4 & $0 \%$ \\
\hline & Type 2 Small & Yes & High & 15 & 68520 & 232180 & 101601 & 0 & 15 & 0 & $0 \%$ & 0 & 0 & 15 & $0 \%$ \\
\hline & $(680 / \mathrm{kg})$ & & Low & 14 & 275640 & 1057073 & 472588 & 0 & 14 & 0 & $0 \%$ & 0 & 1 & 13 & $0 \%$ \\
\hline & & No & High & 5 & 10174 & 152172 & 76818 & 0 & 4 & 1 & $0 \%$ & 2 & 0 & 3 & $40 \%$ \\
\hline & & & Low & 5 & 422392 & 2015326 & 764733 & 0 & 5 & 0 & $0 \%$ & 0 & 1 & 4 & $0 \%$ \\
\hline & Type 1 Large & No & High & 14 & 2572 & 95583 & 6338 & 2 & 12 & 0 & $14 \%$ & 8 & 0 & 6 & $57 \%$ \\
\hline & $(36 / \mathrm{kg})$ & & Low & 8 & 8411 & 174889 & 124725 & 0 & 8 & 0 & $0 \%$ & 2 & 1 & 5 & $25 \%$ \\
\hline & Type 2 Large & Yes & High & 10 & 5887 & 64339 & 29963 & 0 & 10 & 0 & $0 \%$ & 0 & 1 & 9 & $0 \%$ \\
\hline & $(36 / \mathrm{kg})$ & & Low & 10 & 25372 & 227432 & 116941 & 0 & 10 & 0 & $0 \%$ & 0 & 1 & 9 & $0 \%$ \\
\hline
\end{tabular}

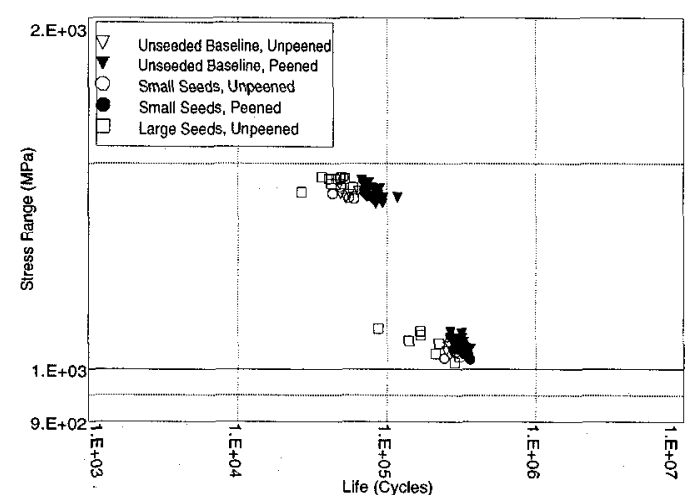

Figure 7: LCF results for Type 1 seeded material (vs. baseline) at $204^{\circ} \mathrm{C}$.

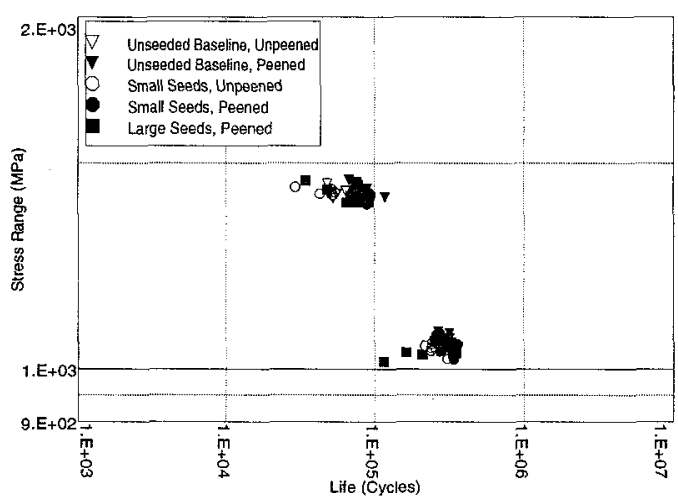

Figure 8: LCF results for Type 2 seeded material (vs. baseline) at $204^{\circ} \mathrm{C}$. 


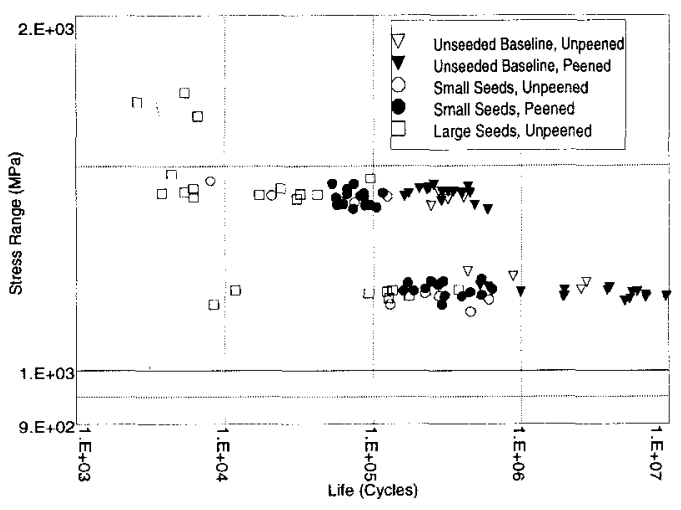

Figure 9: LCF results for Type 1 seeded material (vs. baseline) at $649^{\circ} \mathrm{C}$.

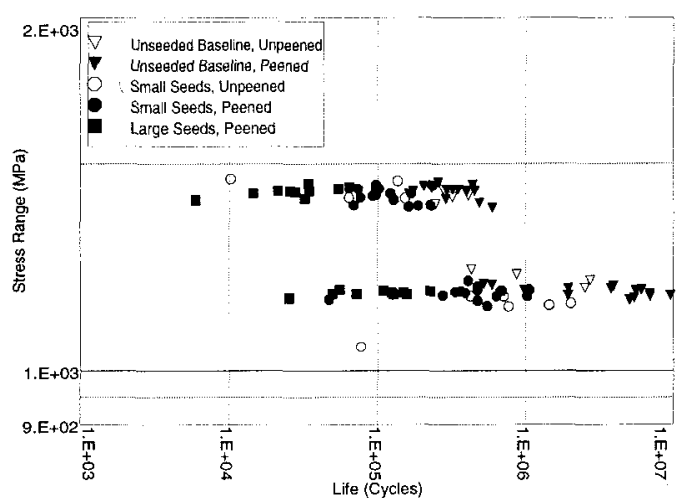

Figure 10: LCF results for Type 2 seeded material (vs. baseline) at $649^{\circ} \mathrm{C}$.

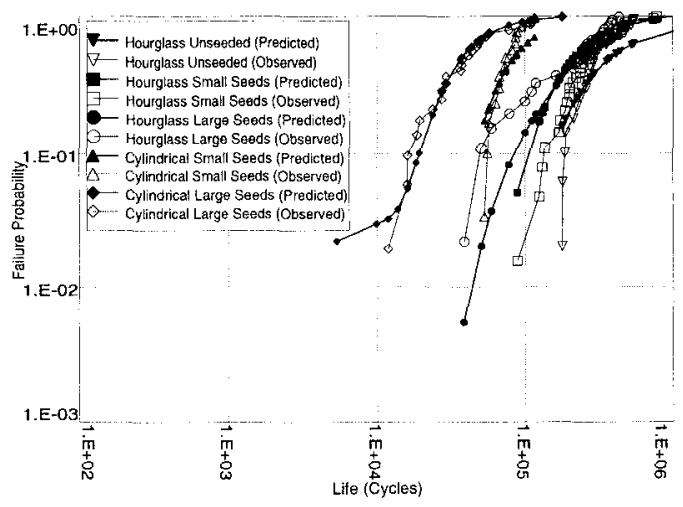

Figure 11: Peened specimens tested at one temperature/strain condition. Comparison of observed vs. probalistically-predicted failure distributions.
Significant efforts are being made to ensure the right inputs. For example, Heavy Liquid Separation (HLS) analysis has been developed to quantify and control inclusion content (Reference 7). Also, the seeded fatigue program discussed in this paper provides a model for crack initiation at inclusions and is continuing.

It is well known that internal initiated failures tend to have longer lives than surface initiated failures, at least at high temperatures (Reference 8). There is a growing body of evidence that buried ceramic inclusions in both P/M Rene' 95 and P/M Rene' 88 DT initiate sharp cracks only very late in life. Figures 12 and 13 compare observed lives with fracture mechanics calculations for both both alloys. Both data sets demonstrate that sharp crack fracture mechanics seriously underestimates total life. To ignore the reality of the incubation phenomenon usually results in very conservative PFM predictions.

It is hypothesized that incubation represents crack initiation in grains near stress concentrating inclusions, and therefore that standard LCF algorithms (e.g. rainflow counting, mean stress corrections and Miner's rule) can be used to calculate incubation given complex cycle missions.

Four points are offered supporting this interpretation:

- While fracture mechanics provides lower bound predictions for most inclusion initiated failures, it is in many cases very conservative (Figures 12 and 13). This is most evidently true for buried inclusions, but it is also true of surface inclusions.

- Given that incubation is observed for inclusions of all sizes, the phenomenon probably cannot be attributed to crack growth threshold.

- It is also observed that incubation life is stress range dependent for the strain controlled tests (again, Figures 12 and 13). This is not the case for the fracture mechanics predictions which are approximately max-stress controlled for negative stress R-ratios.

- There is direct evidence that incubation is a real phenomenon in that most inclusions in a failed fatigue specimen have no readily discernible cracks emanating from them. This is so for both surface intersecting and buried inclusions.

The relative impact of inclusions on LCF life was shown to change dramatically for R $88 \mathrm{DT}$ between $204^{\circ} \mathrm{C}$ and $649^{\circ} \mathrm{C}$. Several factors can be offered to explain the transition: Changes in deformation behavior of the matrix R' $88 \mathrm{DT}$, changes in behavior of the inclusions, or changes in the role of the environment. These factors could work independently or be interrelated.

While the deformation mechanisms of blade superalloys has been well documented, the mechanisms of disk superalloys have not. Waspaloy, which has some similarities to R'88DT, displays planar slip at low temperatures and wavy slip at high temperatures (Reference 9). Planar slip occurs when dislocations tend to be tightly confined to the same plane, where they can combine to form stress concentrations. Wavy slip occurs when thermal activation allows cross-slip onto many planes and results in a more homogeneous dispersal of dislocation damage in the material. For 
a material operating in the planar slip mode, the effect is magnified for larger grain sizes because more dislocations (and higher stress concentration) can be established without the interference of grain boundaries. It is suggested that this is one reason why ASTM 7-8 R'88DT shows such a high percentage of grain failures at low temperatures.

The effect of inclusions at varying temperatures was studied by Jablonski (Reference 5). In this study -500 mesh LC Astroloy was seeded with $\mathrm{Al}_{2} \mathrm{O}_{3}$ (Type 1) and $\mathrm{SiO}_{2}$ (Type 2). The material was tested as HIP'ed (plus heat treated) with a grain size of 30 microns.

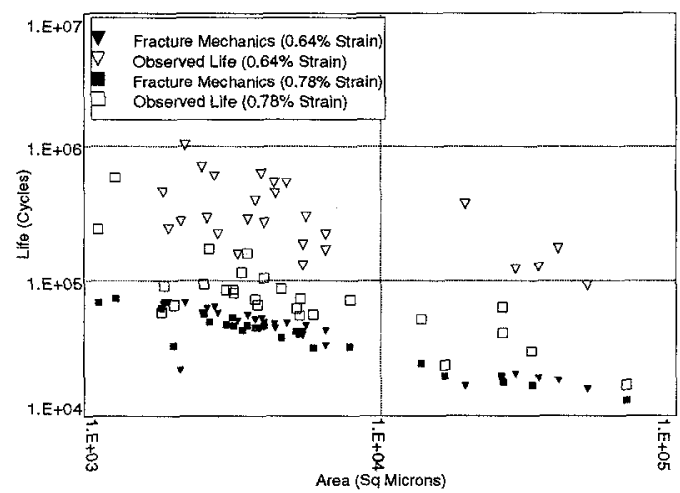

Figure 12: P/M R'88DT -- Fracture mechanics calculations compared to data.

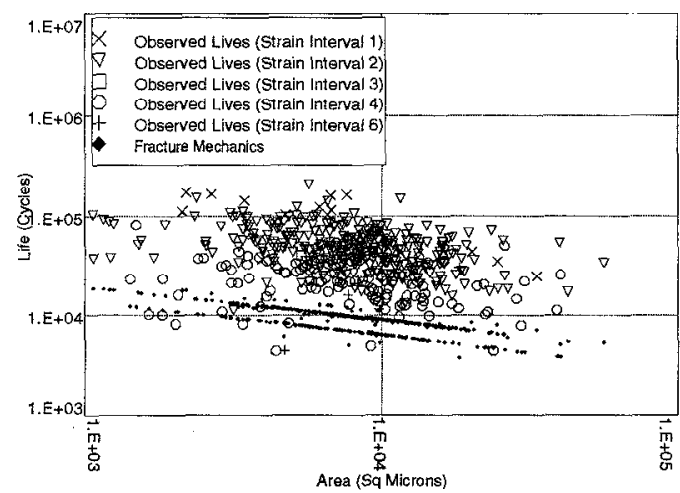

Figure 13: P/M Rene' 95 -- Fracture mechanics calculations compared to data.

The seeds were 75-150 microns in diameter and were added at 4 and $40 \mathrm{ppm}$ for the $\mathrm{Al}_{2} \mathrm{O}_{3}$ and 2 and $20 \mathrm{ppm}$ for the $\mathrm{SiO}_{2}$, based on PFM calculations for the number of seeds required to cause failure. At room temperature, the fractures initiated at the ceramic-metal interface (debonding), and LCF life was not strongly affected by the seeds, but was controlled by Stage I cracking (crystallographic crack propagation along grain facets). At $500^{\circ} \mathrm{C}$, the fractures initiated at cracked seeds, and LCF life was strongly impacted by the seeds. Lives were found to be inversely proportional to stress intensity factors based on seed sizes and distances from the surface.

Jablonski concluded that at low temperatures the inclusions debonded, while at high temperatures they cracked very early in life. It was hypothesized that the cracked inclusions presented much sharper notches and higher stress concentrations than the debonded inclusions. Debonding was not significant in the current study, at either temperature.

Work by Hyzak and coworkers (References $10,11,12$ ) on two P/M alloys AF-115 and AF2-1DA also described a transition in initiation behavior between low and elevated temperatures. These alloys were not seeded, but the AF-115 alloy contained pores, inherent nonmetallic inclusions, and $\mathrm{HfO}_{2}$ particles, and the AF2-1DA alloy contained inherent nonmetallic inclusions.

At elevated temperatures, most initiations occurred at inclusions or pores, while at low temperatures, most initiations were Type 1 grain crystallographic cracking at or near the surface. The data also showed an increased tendency for internal initiation at elevated temperatures, with surface initiations becoming prevalent only at high stresses.

Hyzak explained his results in terms of relative differences in crack initiation vs. propagation as follows:

- At low temperature, planar and heterogeneous slip, given its relative ease in forming at a surface, was always sufficiently pronounced to initiate failure, even if a larger, more angular inclusion may have been present internally.

- At elevated temperature and high stress, cracks were initiated at many stress concentrating pores and smaller ceramics. For cracks at or near the surface, rapid propagation occurred, due to environmental effects and the fact that near-surface flaws have higher stress intensity factors than internal flaws of the same sizes.

- At elevated temperature and low stress, numerous but small surface and near-surface pores and inclusions did not concentrate stress sufficiently to cause initiation, and larger, more angular buried flaws could win the competition for failure.

Obviously the environment must play some role at higher temperatures. However, its exact role is a point of controversy. Hyzak (Reference 12) claimed that the environment promoted rapid crack propagation from surface initiations at high temperature and high stress, but the environment has also been claimed to retard surface crack growth by crack tip blunting by oxide formation (Reference 13). It would be beneficial to study environmental effects further using seeded material and vacuum testing at different frequencies.

The transverse polishing work described above is consistent with Hyzak's discussion. The presence of numerous secondary cracks at $204^{\circ} \mathrm{C}$ probably reflects planar heterogenous slip. With many cracks vying for failure, lower lives and small scatter are not surprising. At $649^{\circ} \mathrm{C}$, the relative absence of secondary cracking (and none in the matrix) probably reflects wavy homogeneous slip. Damage is less likely to accumulate in planes and lead to microcrack formation. Stress concentrating inclusions become more significant. 
Incubation life is found to decrease with increasing inclusion size (Figures 12 and 13). The magnitude of the stress concentration of an inclusion does not directly depend on the size of the inclusion, but the spatial extent of the concentration does. Larger inclusions concentrate more damage than smaller inclusions, leading more quickly to microcrack formation.

While shotpeening was found to be very effective in suppressing surface initiations at both temperatures of the current study, and while it may be presumed that the suppression is due to the residual stresses imparted by the peening (beneficial both for incubation and propagation), the mechanism has not been quantified.

\section{Conclusions}

1. Seeded LCF testing is a powerful technique for studying the impact of ceramic inclusions on P/M alloy life.

2. A $204^{\circ} \mathrm{C}$, for all test conditions, over $80 \%$ of the bars failed at grain facets. Overall, the seeds had very little impact on life, although the large seeded bars did show a smaller percentage of grain facet initiations: For the large Type 1 seeds, about $40 \%$ of the bars failed at seeds, and for the large Type 2 seeds, about $75 \%$ failed at sceds. Sced initiated failures did yicld slightly lower lives.

3. At $204^{\circ} \mathrm{C}$, peening had a negligible effect on LCF life, but it strongly influenced the location of the failures. For unpeened bars, $91.1 \%$ of the bars failed on the surface, while for peened bars, only $4.6 \%$ of the bars failed on the surface. In most cases this resulted in changing the failure site from a surface grain to an internal grain, but the lives were similar regardless of the location.

4. At $649^{\circ} \mathrm{C}$, for all test conditions, the majority of the bars $(98.7 \%)$ failed at inclusions, with life strongly correlated to inclusion size. Unpeened small seeded bars yielded lives $1 / 10$ th to $1 / 4$ those of unpeened unseeded baseline. Peened small seeded bars yielded lives between $1 / 4$ to $1 / 2$ those of unpeened baseline. The lives of the large Type 1 seeded bars, which were all unpeened, were about $6 \%$ those of the unpeened baseline. The fraction was the same for the large Type 2 seeded bars, which were all peened.

5. At $649^{\circ} \mathrm{C}$, peening did appear to suppress surface initiations. For the small seeded material, unpeened bars occasionally failed at surface seeds at high stress, while no peened bars failed on the surface. For the large seeds, all the Type 1 seeded bars were left unpeened, and many failed on the surface. All the Type 2 seeded bars were peened, and all failed either internally or near-surface, but the lives were not improved. The data suggested that Type 2 seeds may be somewhat more deleterious than Type 1 .

6. Internal failures initiated at inclusions at $649^{\circ} \mathrm{C}$ were underpredicted by sharp crack fracture mechanics. It is hypothesized that the incubation prior to sharp crack formation represents fatigue crack initiation in the matrix about the inclusions. This interpretation is the basis of an empirical model which can be used for life prediction. The observations in this paper are consistent with discussions of slip band formation by other authors.

\section{Acknowledgments}

The success of this program was shared as a team effort. The SNECMA team, particularly Pierre-Etienne Mosser, Jean-Yves Guedou, Jean-Charles Lautridou, Gilles Klein, and Jean-Michel Franchez, provided careful material processing. The GEAE team included Tom Knost, Bob Kissinger, Stan Friesen, George Zuefle, Nick Denton, Nancy Sullivan, Paul Domas, Herb Popp, Mike Sauby and Jean Murray Stewart. Don Weaver of Wyman-Gordon Powder Systems (formerly Cameron Powder) produced the R'88DT powder and Denny Dreyer of Special Metals Princeton Operation supplied the material used for the Type 2 seed. Metcut Research Associates, particularly Dave Wingerberg and Bill Stross, provided careful machining and testing support. Careful microscopy by Tom Daniels, Ron Tolhert, Iudy Mescher, Luann Piazza, and Ivan Miller was also appreciated. Finally, the authors wish to thank GEAE for permission to publish this work and the superalloy powder suppliers for their continuous improvements in overall powder cleanliness levels.

\section{References}

1. U. S. Patent No. 4,957,567, D. D. Krueger, R. D. Kissinger, R. G. Menzies, and C. S. Wukusick, General Electric Company.

2. D. D. Krueger, R. D. Kissinger, and R. G. Menzies, "Development and Introduction of a Damage Tolerant High Temperature Nickel-Base Disk Alloy, Rene'88DT," in Superalloys 1992, ed.by S. D. Antolovich et. al, TMS-AIME, Warrendale, PA, 1992, 277-286.

3. D. R. Chang, D. D. Krueger, and R. A. Sprague, Superalloy Powder Processing, Properties, and Turbine Disk Applications, in Superalloys 1984, ed. by M. Gell et. al., TMS-AIME, Warrendale, PA, 1984, .251.

4. E. S. Huron, "Serrated Yielding in a Nickel-Base Superalloy," in Superalloys 1992, ed. by S. D. Antolovich et. al., TMS-AIME, Warrendale, PA, 1992, 675-684.

5. D. A. Jablonski, "The Effect of Ceramic Inclusions on the Low Cycle Fatigue Life of Low Carbon Astroloy Subjected to Hot Isostatic Pressing," Mat. Sci. Eng., 48 (1981), 189-198.

6. P. G. Roth, "Probabilistic Rotor Design System (PRDS) Phase I Interim Report," USAF, Aero Propulsion and Power Directorate, Wright Laboratory, Air Force Systems Command,

Wright-Patterson Air Force Base, Ohio, Report WL-TR-92-2011, (1992).

7. Paul G. Roth, J. C. Murray, J. E. Morra, and J. M. Hyzak, " Heavy Liquid Separation: A Reliable Method to Characterize Inclusions in Metal Powder," Characterization, Testing and Quality Control, Advances in Powder Metallurgy and Particulate Materials, 2, Metal Powder Industries Federation, Princeton, NJ (1994).

8. S. Bashir, P. Taupin, S. D. Antolovich, "Low Cycle Fatigue of As-HIP and HIP+Forged Rene 95," Met. Trans. A, 10A (1979), $1481-1490$ 
9. B. A. Lerch, N. Jayaraman, and S. D. Antolovich; "A Study of Fatigue Damage Mechanisms in Waspaloy from 25 to $800 \mathrm{C}$," Mat. Sci. Eng., 66 (1984), 151-166.

10. J. M. Hyzak and I. M. Bernstein, "The Effect of Defects on the Fatigue Crack Initiation Process in T'wo P/M Superalloys: Part 1. Fatigue Origins," Met. Trans. A, 13A (1982), 33-43.

11. J. M. Hyzak and I. M. Bernstein, "The Effect of Defects on the Fatigue Crack Initiation Process in Two P/M Superalloys: Part 2. Surface-Subsurface Transition," Met. Trans. A, 13A (1982), 45-52.

12. J. M. Hyzak, "The Effect of Defects on the Fatigue Initiation Process in Two P/M Superalloys, AFWAL-TR-80-4063, WPAFB, Ohio, July (1980).

13. L. F. Coffin, Jr., "The Effect of Frequency on the Cyclic Strain and Fatigue Behavior of Cast Rene 80 at 1600F," Met. Trans., 5 (1974), 1053-1060. 